\title{
A GENERALIZATION OF g-SUPPLEMENTED MODULES
}

\author{
BERNA KOŞAR, CELIL NEBIYEV, AND AYTEN PEKIN
}

Received 07 April, 2018

\begin{abstract}
In this work g-radical supplemented modules are defined which generalize gsupplemented modules. Some properties of g-radical supplemented modules are investigated. It is proved that the finite sum of g-radical supplemented modules is g-radical supplemented. It is also proved that every factor module and every homomorphic image of a g-radical supplemented module is g-radical supplemented. Let $R$ be a ring. Then ${ }_{R} R$ is g-radical supplemented if and only if every finitely generated $R$-module is g-radical supplemented. In the end of this work, it is given two examples for g-radical supplemented modules separating with g-supplemented modules.
\end{abstract}

2010 Mathematics Subject Classification: 16D10; 16D70

Keywords: small submodules, radical, supplemented modules, radical (generalized) supplemented modules

\section{INTRODUCTION}

Throughout this paper all rings will be associative with identity and all modules will be unital left modules.

Let $R$ be a ring and $M$ be an $R$-module. We will denote a submodule $N$ of $M$ by $N \leq M$. Let $M$ be an $R$-module and $N \leq M$. If $L=M$ for every submodule $L$ of $M$ such that $M=N+L$, then $N$ is called a small submodule of $M$ and denoted by $N \ll M$. Let $M$ be an $R$-module and $N \leq M$. If there exists a submodule $K$ of $M$ such that $M=N+K$ and $N \cap K=0$, then $N$ is called a direct summand of $M$ and it is denoted by $M=N \oplus K$. For any module $M$, we have $M=M \oplus 0$. $\operatorname{Rad} M$ indicates the radical of $M$. A submodule $N$ of an $R$-module $M$ is called an essential submodule of $M$, denoted by $N \unlhd M$, in case $K \cap N \neq 0$ for every submodule $K \neq 0$. Let $M$ be an $R$-module and $K$ be a submodule of $M . K$ is called a generalized small (briefly, $g$-small) submodule of $M$ if for every $T \unlhd M$ with $M=K+T$ implies that $T=M$, this is written by $K \ll_{g} M$ (in [6], it is called an $e$-small submodule of $M$ and denoted by $K \ll_{e} M$ ). It is clear that every small submodule is a generalized small submodule but the converse is not true generally. Let $M$ be an $R$-module. $M$ is called an hollow module if every proper submodule of $M$ is small in $M . M$ is called a local module if $M$ has the largest submodule, i.e. 
a proper submodule which contains all other proper submodules. Let $U$ and $V$ be submodules of $M$. If $M=U+V$ and $V$ is minimal with respect to this property, or equivalently, $M=U+V$ and $U \cap V \ll V$, then $V$ is called a supplement of $U$ in $M . M$ is called a supplemented module if every submodule of $M$ has a supplement in $M$. Let $M$ be an $R$-module and $U, V \leq M$. If $M=U+V$ and $M=U+T$ with $T \unlhd V$ implies that $T=V$, or equivalently, $M=U+V$ and $U \cap V \ll g M$, then $V$ is called a $g$-supplement of $U$ in $M . M$ is called $g$-supplemented if every submodule of $M$ has a g-supplement in $M$. The intersection of maximal essential submodules of an $R$-module $M$ is called a generalized radical of $M$ and denoted by $\operatorname{Rad}_{g} M$ (in [6], it is denoted by $\operatorname{Rad}_{e} M$ ). If $M$ have no maximal essential submodules, then we denote $\operatorname{Rad}_{g} M=M$.

Lemma $1([2,4,6])$. Let $M$ be an $R$-module and $K, L, N, T \leq M$. Then the followings are hold.

(1) If $K \leq N$ and $N$ is generalized small submodule of $M$, then $K$ is a generalized small submodule of $M$.

(2) If $K$ is contained in $N$ and a generalized small submodule of $N$, then $K$ is a generalized small submodule in submodules of $M$ which contains submodule $N$.

(3) Let $S$ be an $R$-module and $f: M \rightarrow S$ be an $R$-module homomorphism. If $K \ll g M$, then $f(K) \ll_{g} S$.

(4) If $K \ll_{g} L$ and $N \ll_{g} T$, then $K+N \ll_{g} L+T$.

Corollary 1. Let $M_{1}, M_{2}, \ldots, M_{n} \leq M, K_{1} \ll g M_{1}, K_{2} \ll_{g} M_{2}, \ldots, K_{n} \ll g M_{n}$. Then $K_{1}+K_{2}+\ldots+K_{n} \ll g M_{1}+M_{2}+\ldots+M_{n}$.

Corollary 2. Let $M$ be an $R$-module and $K \leq N \leq M$. If $N \ll g M$, then $N / K \ll_{g} M / K$.

Corollary 3. Let $M$ be an $R$-module, $K \ll_{g} M$ and $L \leq M$. Then $(K+L) / L \ll_{g}$ $M / L$.

Lemma 2. Let $M$ be an R-module. Then $\operatorname{Rad}_{g} M=\sum_{L \ll_{g} M} L$.

Proof. See [2].

Lemma 3. The following assertions are hold.

(1) If $M$ is an $R$-module, then $R m \ll_{g} M$ for every $m \in \operatorname{Rad}_{g} M$.

(2) If $N \leq M$, then $\operatorname{Rad}_{g} N \leq \operatorname{Rad}_{g} M$.

(3) If $K, L \leq M$, then $\operatorname{Rad}_{g} K+\operatorname{Rad}_{g} L \leq \operatorname{Rad}_{g}(K+L)$.

(4) If $f: M \longrightarrow N$ is an R-module homomorphism, then $f\left(\operatorname{Rad}_{g} M\right) \leq \operatorname{Rad}_{g} N$.

(5) If $K, L \leq M$, then $\frac{\operatorname{Rad}_{g} K+L}{L} \leq \operatorname{Rad}_{g} \frac{K+L}{L}$.

Proof. Clear from Lemma 1 and Lemma 2.

Lemma 4. Let $M=\oplus_{i \in I} M_{i}$. Then $\operatorname{Rad}_{g} M=\oplus_{i \in I} \operatorname{Rad}_{g} M_{i}$. 
Proof. Since $M_{i} \leq M$, then by Lemma 3(2), $\operatorname{Rad}_{g} M_{i} \leq \operatorname{Rad}_{g} M$ and $\oplus_{i \in I} \operatorname{Rad}_{g} M_{i} \leq \operatorname{Rad}_{g} M$. Let $x \in \operatorname{Rad}_{g} M$. Then by Lemma 3(1), $R x \ll g M$. Since $x \in M=\oplus_{i \in I} M_{i}$, there exist $i_{1}, i_{2}, \ldots, i_{k} \in I$ and $x_{i_{1}} \in M_{i_{1}}, x_{i_{2}} \in M_{i_{2}}, \ldots$, $x_{i_{k}} \in M_{i_{k}}$ such that $x=x_{i_{1}}+x_{i_{2}}+\ldots+x_{i_{k}}$. Since $R x \ll g M$, then by Lemma 1(4), under the canonical epimorphism $\pi_{i_{t}}(t=1,2, \ldots, k) R x_{i_{t}}=\pi_{i_{t}}(R x) \ll g R x_{i_{t}}$. Then $x_{i_{t}} \in \operatorname{Rad}_{g} M_{i_{t}}(t=1,2, \ldots, k)$ and $x=x_{i_{1}}+x_{i_{2}}+\ldots+x_{i_{k}} \in \oplus_{i \in I} \operatorname{Rad}_{g} M_{i}$. Hence $\operatorname{Rad}_{g} M \leq \oplus_{i \in I} \operatorname{Rad}_{g} M_{i}$ and since $\oplus_{i \in I} \operatorname{Rad}_{g} M_{i} \leq \operatorname{Rad}_{g} M, \operatorname{Rad}_{g} M=$ $\oplus_{i \in I} \operatorname{Rad}_{g} M_{i}$.

\section{G-RADICAL SUPPLEMENTED MODULES}

Definition 1. Let $M$ be an $R$-module and $U, V \leq M$. If $M=U+V$ and $U \cap V \leq$ $\operatorname{Rad}_{g} V$, then $V$ is called a generalized radical supplement (briefly, g-radical supplement) of $U$ in $M$. If every submodule of $M$ has a generalized radical supplement in $M$, then $M$ is called a generalized radical supplemented (briefly, g-radical supplemented) module.

Clearly we see that every g-supplemented module is g-radical supplemented. But the converse is not true in general. (See Example 1 and 2.)

Lemma 5. Let $M$ be an $R$-module and $U, V \leq M$. Then $V$ is a g-radical supplement of $U$ in $M$ if and only if $M=U+V$ and $R m \ll g V$ for every $m \in U \cap V$.

Proof. $(\Rightarrow)$ Since $V$ is a g-radical supplement of $U$ in $M, M=U+V$ and $U \cap$ $V \leq \operatorname{Rad}_{g} V$. Let $m \in U \cap V$. Since $U \cap V \leq \operatorname{Rad}_{g} V, m \in \operatorname{Rad}_{g} V$. Hence by Lemma 3(1), $R m \ll_{g} V$.

$(\Leftarrow)$ Since $R m \ll_{g} V$ for every $m \in U \cap V$, then by Lemma 2, $U \cap V \leq \operatorname{Rad}_{g} V$ and hence $V$ is a g-radical supplement of $U$ in $M$.

Lemma 6. Let $M$ be an R-module, $M_{1}, U, X \leq M$ and $Y \leq M_{1}$. If $X$ is a $g$ radical supplement of $M_{1}+U$ in $M$ and $Y$ is a g-radical supplement of $(U+X) \cap$ $M_{1}$ in $M_{1}$, then $X+Y$ is a g-radical supplement of $U$ in $M$.

Proof. Since $X$ is a g-radical supplement of $M_{1}+U$ in $M, M=M_{1}+U+X$ and $\left(M_{1}+U\right) \cap X \leq \operatorname{Rad}_{g} X$. Since $Y$ is a g-radical supplement of $(U+X) \cap M_{1}$ in $M_{1}, M_{1}=(U+X) \cap M_{1}+Y$ and $(U+X) \cap Y=(U+X) \cap M_{1} \cap Y \leq \operatorname{Rad}_{g} Y$. Then $M=M_{1}+U+X=(U+X) \cap M_{1}+Y+U+X=U+X+Y$ and, by Lemma 3(3), $U \cap(X+Y) \leq(U+X) \cap Y+(U+Y) \cap X \leq \operatorname{Rad}_{g} Y+\left(M_{1}+U\right) \cap$ $X \leq \operatorname{Rad}_{g} Y+\operatorname{Rad}_{g} X \leq \operatorname{Rad}_{g}(X+Y)$. Hence $X+Y$ is a g-radical supplement of $U$ in $M$.

Lemma 7. Let $M=M_{1}+M_{2}$. If $M_{1}$ and $M_{2}$ are g-radical supplemented, then $M$ is also g-radical supplemented.

Proof. Let $U \leq M$. Then 0 is a g-radical supplement of $M_{1}+M_{2}+U$ in $M$. Since $M_{1}$ is g-radical supplemented, there exists a g-radical supplement $X$ of 
$\left(M_{2}+U\right) \cap M_{1}=\left(M_{2}+U+0\right) \cap M_{1}$ in $M_{1}$. Then by Lemma $6, X+0=X$ is a g-radical supplement of $M_{2}+U$ in $M$. Since $M_{2}$ is g-radical supplemented, there exists a g-radical supplement $Y$ of $(U+X) \cap M_{2}$ in $M_{2}$. Then by Lemma 6, $X+Y$ is a g-radical supplement of $U$ in $M$.

Corollary 4. Let $M=M_{1}+M_{2}+\ldots+M_{k}$. If $M_{i}$ is g-radical supplemented for every $i=1,2, \ldots, k$, then $M$ is also $g$-radical supplemented.

Proof. Clear from Lemma 7.

Lemma 8. Let $M$ be an $R$-module, $U, V \leq M$ and $K \leq U$. If $V$ is a g-radical supplement of $U$ in $M$, then $(V+K) / K$ is a g-radical supplement of $U / K$ in $M / K$.

Proof. Since $V$ is a g-radical supplement of $U$ in $M, M=U+V$ and $U \cap$ $V \leq \operatorname{Rad}_{g} V$. Then $M / K=U / K+(V+K) / K$ and by Lemma 3(5), $(U / K) \cap$ $((V+K) / K)=(U \cap V+K) / K \leq\left(\operatorname{Rad}_{g} V+K\right) / K \leq \operatorname{Rad}_{g}[(V+K) / K]$. Hence $(V+K) / K$ is a g-radical supplement of $U / K$ in $M / K$.

Lemma 9. Every factor module of a g-radical supplemented module is g-radical supplemented.

Proof. Clear from Lemma 8.

Corollary 5. The homomorphic image of a g-radical supplemented module is $g$ radical supplemented.

Proof. Clear from Lemma 9.

Lemma 10. Let $M$ be a g-radical supplemented module. Then every finitely $M$-generated module is g-radical supplemented.

Proof. Clear from Corollary 4 and Corollary 5.

Corollary 6. Let $R$ be a ring. Then ${ }_{R} R$ is g-radical supplemented if and only if every finitely generated $R$-module is g-radical supplemented.

Proof. Clear from Lemma 10.

Theorem 1. Let $M$ be an $R$-module. If $M$ is g-radical supplemented, then $M / \operatorname{Rad}_{\mathrm{g}} M$ is semisimple.

Proof. Let $U / \operatorname{Rad}_{g} M \leq M / \operatorname{Rad}_{g} M$. Since $M$ is g-radical supplemented, there exists a g-radical supplement $V$ of $U$ in $M$. Then $M=U+V$ and $U \cap V \leq \operatorname{Rad}_{g} V$. Thus $M / \operatorname{Rad}_{g} M=U / \operatorname{Rad}_{g} M+\left(V+\operatorname{Rad}_{g} M\right) / \operatorname{Rad}_{g} M$ and

$$
\begin{aligned}
\left(U / \operatorname{Rad}_{g} M\right) \cap\left(\left(V+\operatorname{Rad}_{g} M\right) / \operatorname{Rad}_{g} M\right) & =\left(U \cap V+\operatorname{Rad}_{g} M\right) / \operatorname{Rad}_{g} M \\
& \leq\left(\operatorname{Rad}_{g} V+\operatorname{Rad}_{g} M\right) / \operatorname{Rad}_{g} M \\
& =\operatorname{Rad}_{g} M / \operatorname{Rad}_{g} M=0 .
\end{aligned}
$$

Hence $M / \operatorname{Rad}_{g} M=U / \operatorname{Rad}_{g} M \oplus\left(V+\operatorname{Rad}_{g} M\right) / \operatorname{Rad}_{g} M$ and $U / \operatorname{Rad}_{g} M$ is a direct summand of $M$. 
Lemma 11. Let $M$ be a g-radical supplemented module and $L \leq M$ with $L \cap$ $\operatorname{Rad}_{g} M=0$. Then $L$ is semisimple. In particular, a g-radical supplemented module $M$ with $\operatorname{Rad}_{g} M=0$ is semisimple.

Proof. Let $X \leq L$. Since $M$ is g-radical supplemented, there exists a g-radical supplement $T$ of $X$ in $M$. Hence $M=X+T$ and $X \cap T \leq \operatorname{Rad}_{g} T \leq \operatorname{Rad}_{g} M$. Since $M=X+T$ and $X \leq L$, by Modular Law, $L=L \cap M=L \cap(X+T)=X+$ $L \cap T$. Since $X \cap T \leq \operatorname{Rad}_{g} M$ and $L \cap \operatorname{Rad}_{g} M=0, X \cap L \cap T=L \cap X \cap T \leq$ $L \cap \operatorname{Rad}_{g} M=0$. Hence $L=X \oplus L \cap T$ and $X$ is a direct summand of $L$.

Proposition 1. Let $M$ be a g-radical supplemented module. Then $M=K \oplus L$ for some semisimple module $K$ and some module $L$ with essential generalized radical.

Proof. Let $K$ be a complement of $\operatorname{Rad}_{g} M$ in $M$. Then by [5, 17.6], $K \oplus \operatorname{Rad}_{g} M \unlhd M$. Since $K \cap \operatorname{Rad}_{g} M=0$, then by Lemma $11, K$ is semisimple. Since $M$ is g-radical supplemented, there exists a g-radical supplement $L$ of $K$ in $M$. Hence $M=K+L$ and $K \cap L \leq \operatorname{Rad}_{g} L \leq \operatorname{Rad}_{g} M$. Then by $K \cap \operatorname{Rad}_{g} M=0$, $K \cap L=0$. Hence $M=K \oplus L$. Since $M=K \oplus L$, then by Lemma $4, \operatorname{Rad}_{g} M=$ $\operatorname{Rad}_{g} K \oplus \operatorname{Rad}_{g} L$. Hence $K \oplus \operatorname{Rad}_{g} M=K \oplus \operatorname{Rad}_{g} L$. Since $K \oplus \operatorname{Rad}_{g} L=$ $K \oplus \operatorname{Rad}_{g} M \unlhd M=K \oplus L$, then by [1, Proposition 5.20], $\operatorname{Rad}_{g} L \unlhd L$.

Proposition 2. Let $M$ be an $R-$ module and $U \leq M$. The following statements are equivalent.

(1) There is a decomposition $M=X \oplus Y$ with $X \leq U$ and $U \cap Y \leq \operatorname{Rad}_{g} Y$.

(2) There exists an idempotent $e \in$ End $(M)$ with $e(M) \leq U$ and $(1-e)(U) \leq$ $\operatorname{Rad}_{g}(1-e)(M)$.

(3) There exists a direct summand $X$ of $M$ with $X \leq U$ and $U / X \leq \operatorname{Rad}_{g}(M / X)$.

(4) $U$ has a g-radical supplement $Y$ such that $U \cap Y$ is a direct summand of $U$.

Proof. (1) $\Rightarrow$ (2) For a decomposition $M=X \oplus Y$, there exists an idempotent $e \in \operatorname{End}(M)$ with $X=e(M)$ and $Y=(1-e)(M)$. Since $e(M)=X \leq U$, we easily see that $(1-e)(U)=U \cap(1-e)(M)$. Then by $Y=(1-e)(M)$ and $U \cap Y \leq$ $\operatorname{Rad}_{g} Y,(1-e)(U)=U \cap(1-e)(M)=U \cap Y \leq \operatorname{Rad}_{g} Y=\operatorname{Rad}_{g}(1-e)(M)$.

(2) $\Rightarrow$ (3) Let $X=e(M)$ and $Y=(1-e)(M)$. Since $e \in E n d(M)$ is idempotent, we easily see that $M=X \oplus Y$. Then $M=U+Y$. Since $e(M)=X \leq U$, we easily see that $(1-e)(U)=U \cap(1-e)(M)$. Since $M=U+Y$ and $U \cap Y=$ $U \cap(1-e)(M)=(1-e)(U) \leq \operatorname{Rad}_{g}(1-e)(M)=\operatorname{Rad}_{g} Y, Y$ is a g-radical supplement of $U$ in $M$. Then by Lemma $8, M / X=(Y+X) / X$ is a g-radical supplement of $U / X$ in $M / X$. Hence $U / X=(U / X) \cap(M / X) \leq \operatorname{Rad}_{g}(M / X)$.

(3) $\Rightarrow$ (4) Let $M=X \oplus Y$. Since $X \leq U, M=U+Y$. Let $t \in U \cap Y$ and $R t+T=Y$ for an essential submodule $T$ of $Y$. Let $((T+X) / X) \cap(L / X)=0$ for a submodule $L / X$ of $M / X$. Then $(L \cap T+X) / X=((T+X) / X) \cap(L / X)=0$ and $L \cap T+X=X$. Hence $L \cap T \leq X$ and since $X \cap Y=0, L \cap T \cap Y \leq X \cap Y=0$. Since $L \cap Y \cap T=L \cap T \cap Y=0$ and $T \unlhd Y, L \cap Y=0$. Since $X \leq L$ and 
$M=X+Y$, by Modular Law, $L=L \cap M=L \cap(X+Y)=X+L \cap Y=X+0=$ $X$. Hence $L / X=0$ and $(T+X) / X \unlhd M / X$. Since $R t+T=Y, R(t+X)+$ $(T+X) / X=(R t+X) / X+(T+X) / X=(R t+T+X) / X=(Y+X) / X=$ $M / X$. Since $t \in U, t+X \in U / X \leq \operatorname{Rad}_{g}(M / X)$ and hence $R(t+X) \ll g M / X$. Then by $R(t+X)+(T+X) / X=M / X$ and $(T+X) / X \unlhd M / X,(T+X) / X=$ $M / X$ and then $X+T=M$. Since $X+T=M$ and $T \leq Y$, by Modular Law, $Y=Y \cap M=Y \cap(X+T)=X \cap Y+T=0+T=T$. Hence $R t \ll g Y$ and by Lemma 5, $Y$ is a g-radical supplement of $U$ in $M$. Since $M=X \oplus Y$ and $X \leq U$, by Modular Law, $U=U \cap M=U \cap(X \oplus Y)=X \oplus U \cap Y$. Hence $U \cap Y$ is a direct summand of $U$.

(4) $\Rightarrow$ (1) Let $U=X \oplus U \cap Y$ for a submodule $X$ of $U$. Since $Y$ is a g-radical supplement of $U$ in $M, M=U+Y$ and $U \cap Y \ll g Y$. Hence $M=U+Y=$ $(X \oplus U \cap Y)+Y=X \oplus Y$.

Lemma 12. Let $V$ be a g-radical supplement of $U$ in $M$. If $U$ is a generalized maximal submodule of $M$, then $U \cap V$ is a unique generalized maximal submodule of $V$.

Proof. Since $U$ is a generalized maximal submodule of $M$ and $V /(U \cap V) \simeq$ $(V+U) / U=M / U, U \cap V$ is a generalized maximal submodule of $V$. Hence $\operatorname{Rad}_{g} V \leq U \cap V$ and since $U \cap V \leq \operatorname{Rad}_{g} V, \operatorname{Rad}_{g} V=U \cap V$. Thus $U \cap V$ is a unique generalized maximal submodule of $V$.

Definition 2. Let $M$ be an $R$-module. If every proper essential submodule of $M$ is generalized small in $M$ or $M$ has no proper essential submodules, then $M$ is called a generalized hollow module.

Clearly we see that every hollow module is generalized hollow.

Definition 3. Let $M$ be an $R$-module. If $M$ has a large proper essential submodule which contain all essential submodules of $M$ or $M$ has no proper essential submodules, then $M$ is called a generalized local module.

Clearly we see that every local module is generalized local.

Proposition 3. Let $M$ be an $R$-module and $\operatorname{Rad}_{g} M \neq M$. Then $M$ is generalized hollow if and only if $M$ is generalized local.

Proof. ( $\Longrightarrow$ ) Let $M$ be generalized hollow and let $L$ be a proper essential submodule of $M$. Then $L \ll_{g} M$ and by Lemma $2, L \leq \operatorname{Rad}_{g} M$. Thus $\operatorname{Rad}_{g} M$ is a proper essential submodule of $M$ which contain all proper essential submodules of M.

$(\Longleftarrow$ ) Let $M$ be a generalized local module, $T$ be the largest proper essential submodule of $M$ and $L$ be a proper essential submodule of $M$. Let $L+S=M$ with $S \unlhd M$. If $S \neq M$, then $L+S \leq T \neq M$. Thus $S=M$ and $L \ll g M$. 
Definition 4. Let $M$ be an $R$-module and $U, V \leq M$. If $M=U+V$ and $U \cap$ $V \ll_{g} M$, then $V$ is called a weak g-supplement of $U$ in $M$. If every submodule of $M$ has a weak g-supplement in $M$, then $M$ is called a weakly g-supplemented module. (See [3]).

Clearly we can see that if $M$ is a weakly g-supplemented module, then $M$ is gsemilocal $\left(M / \operatorname{Rad}_{g} M\right.$ is semisimple, see [3]).

Proposition 4. Generalized hollow and generalized local modules are weakly gsupplemented, so are g-semilocal.

Proof. Clear from definitions.

Proposition 5. Let $M$ be a g-radical supplemented module with $\operatorname{Rad}_{g} M \ll_{g} M$. Then $M$ is weakly g-supplemented.

Proof. Clear from definitions.

Example 1. Consider the $\mathbb{Z}$-module $\mathbb{Q}$. Since $\operatorname{Rad}_{g} \mathbb{Q}=\operatorname{Rad} \mathbb{Q}=\mathbb{Q}, \mathbb{Z} \mathbb{Q}$ is g-radical supplemented. But, since $\mathbb{Z} \mathbb{Q}$ is not supplemented and every nonzero submodule of $\mathbb{Z} \mathbb{Q}$ is essential in $\mathbb{Z} \mathbb{Q}, \mathbb{Z} \mathbb{Q}$ is not $\mathrm{g}$-supplemented.

Example 2. Consider the $\mathbb{Z}$-module $\mathbb{Q} \oplus \mathbb{Z}_{p^{2}}$ for a prime $p$. It is easy to check that $\operatorname{Rad}_{g} \mathbb{Z}_{p^{2}} \neq \mathbb{Z}_{p^{2}}$. By Lemma $4, \operatorname{Rad}_{g}\left(\mathbb{Q} \oplus \mathbb{Z}_{p^{2}}\right)=\operatorname{Rad}_{g} \mathbb{Q} \oplus \operatorname{Rad}_{g} \mathbb{Z}_{p^{2}} \neq$ $\mathbb{Q} \oplus \mathbb{Z}_{p^{2}}$. Since $\mathbb{Q}$ and $\mathbb{Z}_{p^{2}}$ are g-radical supplemented, by Lemma 7, $\mathbb{Q} \oplus \mathbb{Z}_{p^{2}}$ is g-radical supplemented. But $\mathbb{Q} \oplus \mathbb{Z}_{p^{2}}$ is not g-supplemented.

\section{REFERENCES}

[1] F. W. Anderson and K. R. Fuller, Rings and Categories of Modules (Graduate Texts in Mathematics). New York: Springer, 1998.

[2] B. Koşar, C. Nebiyev, and N. Sökmez, "G-supplemented modules," Ukrainian Mathematical Journal, vol. 67, no. 6, pp. 861-864, 2015, doi: 10.1007/s11253-015-1127-8.

[3] C. Nebiyev and H. H. Ökten, "Weakly g-supplemented modules," European Journal of Pure and Applied Mathematics, vol. 10, no. 3, pp. 521-528, 2017.

[4] N. Sökmez, B. Koşar, and C. Nebiyev, "Genelleştirilmiş küçük alt modüller," in XIII. Ulusal Matematik Sempozyuтu. Kayseri: Erciyes Üniversitesi, 2010.

[5] R. Wisbauer, Foundations of Module and Ring Theory. Philadelphia: Gordon and Breach, 1991.

[6] D. X. Zhou and X. R. Zhang, "Small-essential submodules and morita duality," Southeast Asian Bulletin of Mathematics, vol. 35, pp. 1051-1062, 2011.

Authors' addresses

Berna Koşar

Department of Mathematics, Ondokuz Mayıs University, 55270, Kurupelit-Atakum, Samsun, Turkey

E-mail address: bernak@omu.edu.tr 


\section{Celil Nebiyev}

Department of Mathematics, Ondokuz Mayıs University, 55270, Kurupelit-Atakum, Samsun, Turkey

E-mail address: cnebiyev@omu.edu.tr

Ayten Pekin

Department of Mathematics, İstanbul University, İstanbul, Turkey

E-mail address: aypekin@istanbul.edu.tr 